\title{
Adaptive Hash Routing for a Cluster of Client-Side Web Proxies
}

\author{
Xueyan Tang \\ School of Computer Engineering \\ Nanyang Technological University \\ Singapore 639798 \\ asxytang@ntu.edu.sg
}

\author{
Samuel T. Chanson \\ Department of Computer Science \\ The Hong Kong University of Science and Technology \\ Clear Water Bay, Hong Kong \\ chanson@cs.ust.hk
}

\begin{abstract}
Hash routing reduces cache misses for a cluster of web proxies by eliminating the duplication of cache contents. In this paper, we investigate the optimization of hash routing performance by dynamically adapting object and DNS allocations to the traffic pattern. An analytical model is developed for hash routing that takes into consideration the original request distribution, the object allocation strategy, the speeds of the proxies and the cache hit ratios. Based on this model, the optimal hash routing problem is studied. The analytical results are applied to the design of two adaptive hash routing schemes: ADA-OBJ optimizes object allocation under static client configuration, and ADA-OBJ/DNS optimizes both object and DNS allocations under dynamic client configuration. Trace-driven simulation experiments have been conducted to evaluate the performance of the proposed schemes. The results show that they significantly outperform the intuitive static hash routing scheme based only on the speeds of the proxies.
\end{abstract}

Keywords: cooperative web caching, hash routing, object allocation, DNS allocation, load distribution, performance analysis, non-linear optimization

\section{Introduction}

The explosive growth of the World Wide Web has resulted in tremendous increase in Internet traffic. Proxy caching has been recognized as an important technique to improve web performance $[1,2,3,4,5]$. Web proxies [6] are often located between the clients and origin servers. They cache frequently accessed web objects at points close to the clients. This has the benefits of reducing network traffic and alleviating server load at the origin servers, thereby decreasing the download time of web objects.

To handle high volume of concurrent requests, a cluster of web proxies connected by a high speed local area network is often set up in the client organization (e.g., a corporation or a university) [7]. The cluster needs a 
coordinating protocol to obtain the full benefits of caching. Since the proxies have similar network distances to the origin servers, the main objective of coordination is to improve the overall hit ratio and reduce the outbound traffic. Hash routing is a technique with this objective in mind $[7,8]$. In this approach, a hash function maps web objects (i.e., their URLs) to a hash space which is in turn partitioned among the proxies. Each proxy is responsible for the objects in its assigned partition. Each client is associated with a proxy known as its configured proxy. This is done by statically configuring the client browser (static client configuration) or by dynamically allocating the client to one of the proxies via a domain name server (DNS) at the time of object requests (dynamic client configuration) [4]. To retrieve an object, the client sends the request to its configured proxy (see step ' $a$ ' in Figure 1). The configured proxy hashes the URL of the requested object, determines the hashed proxy to which the object is assigned, and sends the request to the hashed proxy on behalf of the client (step ' $b$ '). If the requested object is cached in the hashed proxy, the object is sent to the configured proxy. Otherwise, the hashed proxy fetches the object from the origin server (step 'c'), places a copy in its cache if desired, and forwards the object to the configured proxy. Inter-proxy communication is not required if the configured and hashed proxies are the same. On receiving the object, the configured proxy sends it to the client but does not put a copy in its cache. In hash routing, at most one copy of each object is stored in the proxy cluster, thereby improving the overall hit ratio. Hash routing also reduces the overhead of locating objects compared to the other cooperative caching approaches such as sending broadcast queries and maintaining directories $[9,10,11]$.

The approach described above is called proxy-executed hash routing (proxy-HR). An alternative called clientexecuted hash routing (client-HR) is to have the client compute the hash function and send the request to the hashed proxy directly. However, there are several weaknesses with client-HR that make it less widely applicable than proxy-HR. From the administrator's perspective, client-HR requires software modification in all client machines and hence is more complicated to deploy than proxy-HR [7]. Moreover, in client-HR, it is difficult to update the hash function at each client when the function changes (e.g., when proxies are added or removed). In addition, some browsers may not have hashing capabilities and it is not desirable to perform hashing on thin clients such as mobile devices. Furthermore, there exist environments where the clients are not allowed to directly contact all the proxies. For example, in the mobile computing environment, a client can only communicate with the proxy located in its current vicinity through wireless networks $[12,13]$. Therefore, we shall consider proxy-HR only in this work.

Appropriately distributing web objects and mapping DNS queries to the proxies are crucial to hash routing performance. However, they are challenging tasks. First, as web workload changes with time, static object and DNS allocations would not work well. It is desirable to dynamically adapt both allocation strategies to the traffic pattern. Second, clients normally work independently of one another. Therefore, the client to proxy association and hence the request distribution among the proxies can be highly skewed under static client configuration, leading to significant load imbalance. Moreover, due to periodic and incremental facility upgrade, the proxies 


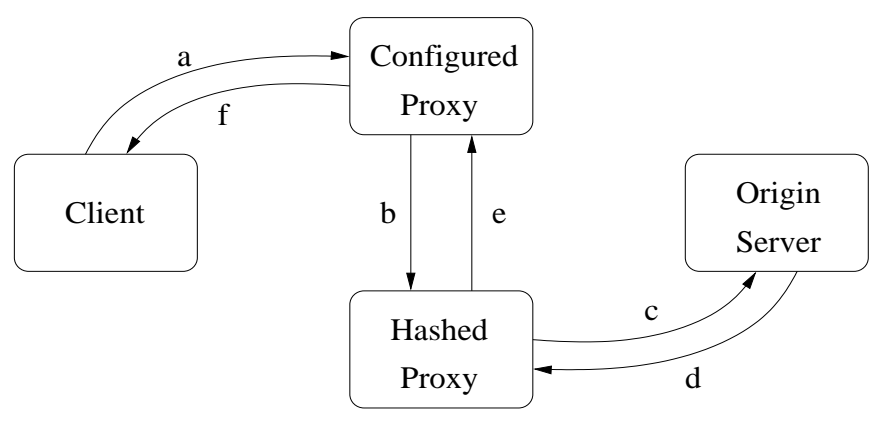

a: request object from configured proxy

b: request object from hashed proxy\#

c: request object from origin server*

$\mathrm{d}$ : send object to hashed proxy*

e: send object to configured proxy\#

f: send object to client

\# omitted if the configured proxy is the same as the hash proxy

* omitted if the object is in the cache of the hashed proxy

Figure 1: Hash Routing among Web Proxies

may have different processing speeds and storage capacities. Research on distributed systems has shown that simple speed-proportional workload allocation does not produce optimal performance if the servers have different speeds [14]. Thus, both object and DNS allocations should be optimized since they directly affect the distribution of workload among the proxies. Finally, the impact of object caching should be taken into consideration. To the best of our knowledge, there has been no systematic study on hash routing among web proxies that addresses the above issues.

This paper investigates the optimization of object and DNS allocations to improve the performance of hash routing. We first develop a general analytical model for hash routing that takes into consideration the important factors described above: the request distribution, the object to proxy allocation, the speed heterogeneity of proxies, the cache hit ratio, and their interaction effects. Based on this model, the problem of determining the optimal object and DNS allocations to minimize the steady-state response time of requests is investigated (we shall refer to it as the optimal hash routing problem). The analytical results are applied to the design of adaptive hash routing schemes for different client configurations, and the performance impacts of various system parameters are studied by trace-based simulation experiments. The results show that the proposed adaptive schemes significantly reduce load imbalance among the proxies and outperform the intuitive static hash routing scheme based only on proxy speeds.

The rest of this paper is organized as follows. Section 2 presents an analytical model for hash routing. Section 3 investigates the optimal hash routing problems for static and dynamic client configurations, and proposes two adaptive hash routing schemes. Section 4 describes the simulation setup. The experimental results are presented and discussed in Section 5. Section 6 summarizes the related work and finally, Section 7 concludes the paper. 


\section{Analytical Model for Hash Routing}

In this section, we present an analytical model for hash routing. For clarity of presentation, the requests sent from a client to its configured proxy are called original requests (step ' $a$ ' in Figure 1); the requests sent from a configured proxy to a hashed proxy are called hashed requests (step 'b'); and the requests sent from a hashed proxy to an origin server are called remote requests (step ' $c$ ').

\subsection{System Model}

We consider a cluster of $n$ web proxies $P_{1}, P_{2}, \ldots, P_{n}$ serving a large group of clients (see Figure 2). The proxies are assumed, as in actual practice, to be located much closer to the clients than to the origin servers. Each proxy $P_{i}$ has a request processing rate of $s_{i} \mu$, where $\mu$ is the baseline rate and $s_{i}$ is the relative speed of $P_{i}$. The mean and mean-square request processing times on a baseline proxy are denoted by $p=1 / \mu$ and $J$ respectively. Each proxy $P_{i}$ has a cache size of $C_{i}$. Let $S$ be the average size of web objects. The mean number of objects that can be cached in $P_{i}$ is given by $c_{i}=C_{i} / S$.

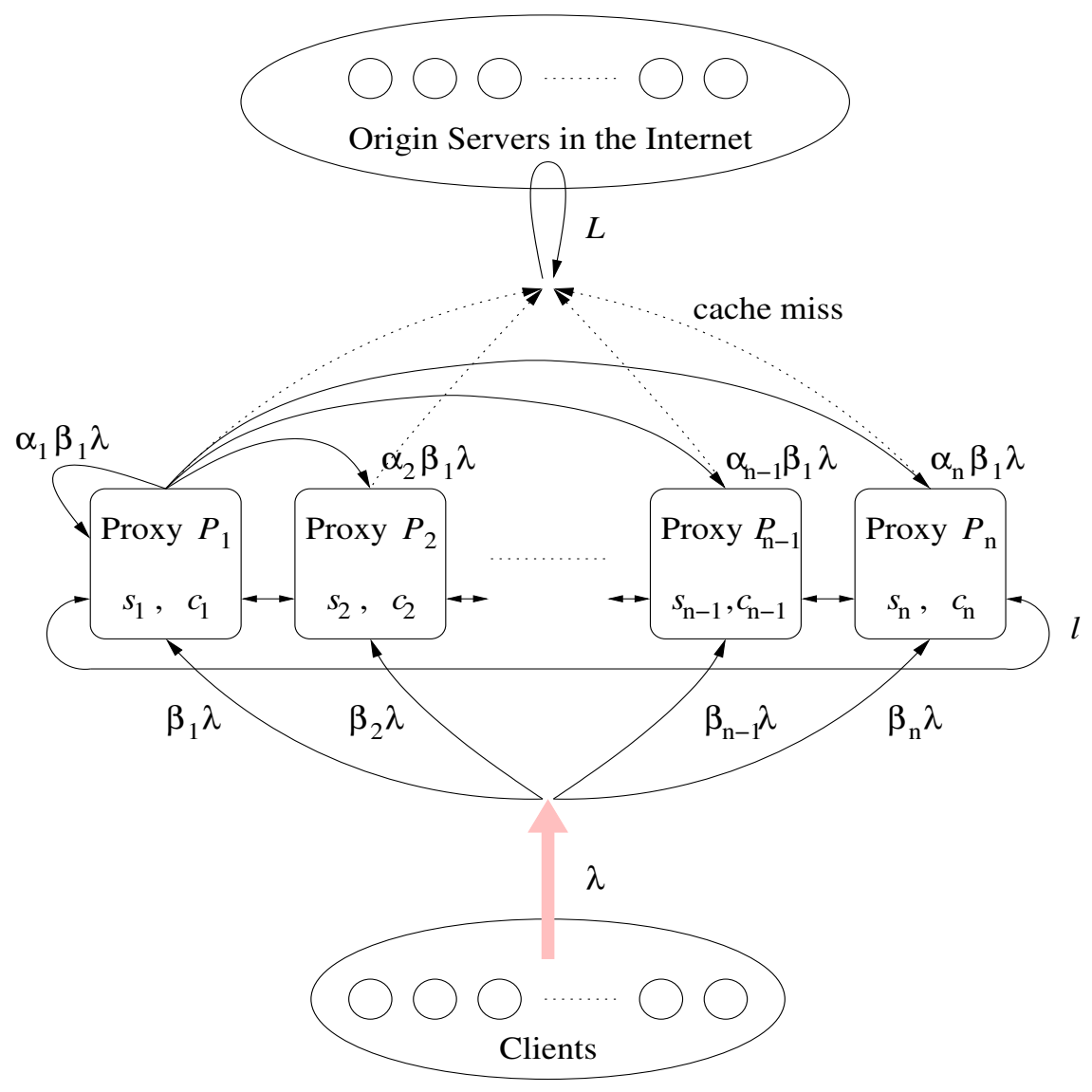

Figure 2: System Model

The aggregate arrival rate of original requests from all clients is denoted by $\lambda$. A fraction $\beta_{i}$ of the original 
requests are initially sent to $P_{i}$ as their configured proxy based on static or dynamic client configuration, where $0 \leq \beta_{i} \leq 1$ and $\sum_{i=1}^{n} \beta_{i}=1$. The hash routing scheme allocates a fraction $\alpha_{i}$ of all objects to proxy $P_{i}$, where $0 \leq \alpha_{i} \leq 1$ and $\sum_{i=1}^{n} \alpha_{i}=1$. This can be done by first mapping web objects to a hash space (e.g., an integer in the range from 0 to $2^{31}-1$, or an $n$-dimension vector where $n$ is the number of proxies as in the example hash function described in Section 4.4). A fraction $\alpha_{i}$ of the hash space is then assigned to proxy $P_{i}$ [15]. We shall refer to the vector $\left(\alpha_{1}, \alpha_{2}, \ldots, \alpha_{n}\right)$ as the object allocation strategy. Note that the proxies maintain the object to proxy allocation in the form of a hash function. They are not required to be aware of the total object set available on the web. It is assumed that a fraction $\alpha_{i}$ of the original requests are directed to the objects allocated to $P_{i}$. This assumption is reasonable because the number of objects accessed by the clients in an organization is much larger (e.g., 5 to 6 orders of magnitude higher) than the number of proxies. Therefore, the fraction of requests hashed to each proxy depends heavily on the number of objects in its assigned partition rather than the individual access rates of the objects [15]. Furthermore, we assume the hash allocation and the initial distribution of original requests to the proxies are independent. That is, a fraction $\alpha_{i} \beta_{j}$ of the original requests are initially sent to $P_{j}$ but refer to objects allocated to $P_{i}$.

The average network delay of inter-proxy communication is denoted by $l$. For each cache miss, an average delay $L$ is needed to fetch the object from the origin server which can be quite far away from the proxy. ${ }^{1}$ Since the proxies in the cluster are situated in close network proximity, $L$ is usually much larger than $l(L \gg l)$.

Table 1 summarizes the notations used in our analysis.

Table 1: Summary of Notations

\begin{tabular}{|c|l|}
\hline Notation & Definition \\
\hline$\mu$ & baseline request processing rate \\
$p$ & mean request processing time on a baseline proxy \\
$J$ & mean-square request processing time on a baseline proxy \\
$s_{i}$ & relative processing speed of proxy $P_{i}$ \\
$S$ & average object size \\
$C_{i}$ & cache size of proxy $P_{i}$ \\
$c_{i}$ & mean number of objects that can be cached in proxy $P_{i}$ \\
$\alpha_{i}$ & fraction of objects allocated to proxy $P_{i}$ \\
$\beta_{i}$ & fraction of original requests initially sent to proxy $P_{i}$ \\
$l$ & average inter-proxy communication delay \\
$L$ & average cache miss delay \\
\hline
\end{tabular}

\footnotetext{
${ }^{1} L$ includes processing delay at the origin server as well as network delay.
} 


\subsection{Performance Analysis}

The performance metric in our analysis is the average response time of original requests. The response time is defined as the delay from the time the request arrives at the configured proxy to the time the configured proxy finishes sending the entire object to the client. ${ }^{2}$ It includes queueing and processing delays at the proxies, network transfer delays between the proxies, and possible cache miss delays to fetch the object from the origin server.

We start by computing the cache hit ratio at each proxy. The cache hit ratio of proxy $P_{i}$ is defined as the ratio of the number of requests satisfied by $P_{i}$ 's cache to the total number of requests hashed to $P_{i}$. Note that this ratio is independent of the original requests that are initially sent to $P_{i}$ but hashed to other proxies.

Each cached object $O$ reduces the network traffic between the proxy and the origin servers by $f(O) \cdot s(O)$, where $f(O)$ and $s(O)$ are the access frequency and the size of $O$ respectively. The problem of filling the cache to maximize the overall reduction in network traffic is equivalent to the knapsack problem which is NPcomplete [16]. A fast greedy heuristic is to cache objects in decreasing order of access frequency until the cache space is filled up. This is called the perfect LFU replacement algorithm and is the best online algorithm under an independent reference model [17]. An approximate implementation of this algorithm is described in the experimental setup in Section 4.3.

It is known that the access frequencies of web objects follow a Zipf-like distribution [17], i.e., the access frequency of the $i$ th most popular object is proportional to $1 / i^{\theta}$, where $0<\theta<1$. For simplicity, the requests hashed to each proxy are assumed to follow the Zipf-like distribution with the same parameter $\theta$ (this assumption and the following calculation method of cache hit ratio are validated by trace-based simulation experiments in Section 5.1). Let $N$ be the total number of objects, then the cache hit ratio of proxy $P_{i}$ is given by

$$
\begin{aligned}
H\left(P_{i}\right) & =\frac{1^{-\theta}+2^{-\theta}+\cdots+c_{i}^{-\theta}}{1^{-\theta}+2^{-\theta}+\cdots+\left(\alpha_{i} N\right)^{-\theta}} \\
& \approx \frac{\int_{0}^{c_{i}} x^{-\theta} d x}{\int_{0}^{\alpha_{i} N} x^{-\theta} d x}=\frac{\frac{1}{1-\theta} c_{i}^{1-\theta}}{\frac{1}{1-\theta}\left(\alpha_{i} N\right)^{1-\theta}}=\frac{c_{i}^{1-\theta}}{\left(\alpha_{i} N\right)^{1-\theta}}=\frac{c_{i}^{1-\theta}}{\alpha_{i}^{1-\theta}} \cdot E,
\end{aligned}
$$

where $E=1 / N^{1-\theta}$ is a constant. Note that the approximation is from the Riemann sum of integration.

Our analysis is conducted under the assumption of Poisson arrivals, which has been shown to match random arrivals reasonably well in various contexts $[18,19]$. The adaptive hash routing schemes are proposed based on the analysis. In the experiments, we tested the proposed schemes with real web traffic. As will be shown by the experimental results in Section 5, the above assumption is sufficient for the purpose of computing optimized object allocation strategies. Our adaptive hash routing schemes significantly outperform the static speed-based hash routing scheme (see Section 5).

\footnotetext{
${ }^{2}$ The network delay from the client to the configured proxy is independent of the hash routing scheme. For simplicity, this delay is not included in the response time.
} 
Each proxy is modeled as an $M / G / 1$ queueing system employing the First-Come-First-Serve (FCFS) scheduling discipline. The queueing system serves incoming requests from the clients and the other proxies. To compute the queueing delay at each proxy, we first calculate the arrival rate of incoming requests as well as their mean and mean-square service times at proxy $P_{i}$. Due to object caching and hash routing, an additional request may have to be issued by $P_{i}$ to assist the completion of an incoming request. The service time of an incoming request is defined as the total processing time of $P_{i}$ spent in satisfying the request. Note that the incoming requests can be classified into three categories based on how they are handled by $P_{i}$ :

1. Requests resulting in cache hits

Cache hits can result from two types of requests: (1) hashed requests issued by the configured proxies of original requests whose configured and hashed proxies are different; and (2) original requests whose configured and hashed proxies are the same. The total arrival rate of these two types of requests is $\left(\sum_{j \neq i} \alpha_{i} \beta_{j} \lambda\right)+\alpha_{i} \beta_{i} \lambda=\alpha_{i} \lambda$. Thus, the arrival rate of requests resulting in cache hits is given by $H\left(P_{i}\right) \cdot \alpha_{i} \lambda=\alpha_{i}^{\theta} c_{i}^{1-\theta} E \lambda$. Since there is no need for $P_{i}$ to contact other proxies or origin servers to satisfy these requests, the average service time is $p / s_{i}=1 /\left(s_{i} \mu\right)$ and the mean-square service time is $J / s_{i}^{2}$.

2. Requests resulting in cache misses

Cache misses can result from the same two types of requests as in the first category. Hence, the arrival rate of requests resulting in cache misses is given by $\left(1-H\left(P_{i}\right)\right) \cdot \alpha_{i} \lambda=\left(\alpha_{i}-\alpha_{i}^{\theta} c_{i}^{1-\theta} E\right) \lambda$. Since a remote request is sent by $P_{i}$ to fetch the requested object from the origin server, $P_{i}$ needs to process two requests for each incoming request. Therefore, the average service time is $2 p / s_{i}=2 /\left(s_{i} \mu\right)$, and the mean-square service time is $4 J / s_{i}^{2}$.

3. Requests that are hashed to other proxies

Original requests whose configured and hashed proxies are different fall into this category. The arrival rate of these requests is $\beta_{i} \lambda-\alpha_{i} \beta_{i} \lambda=\left(1-\alpha_{i}\right) \beta_{i} \lambda$. Since a hashed request is sent by $P_{i}$ to the hashed proxy, similar to the second category, $P_{i}$ needs to process two requests for each incoming request. Thus, the average service time is $2 p / s_{i}=2 /\left(s_{i} \mu\right)$, and the mean-square service time is $4 J / s_{i}^{2}$.

Therefore, the total arrival rate of incoming requests at proxy $P_{i}$ is

$$
\begin{aligned}
\lambda_{i} & =\alpha_{i}^{\theta} c_{i}^{1-\theta} E \lambda+\left(\alpha_{i}-\alpha_{i}^{\theta} c_{i}^{1-\theta} E\right) \lambda+\left(1-\alpha_{i}\right) \beta_{i} \lambda \\
& =\left(\alpha_{i}+\beta_{i}-\alpha_{i} \beta_{i}\right) \lambda=B_{i} \lambda,
\end{aligned}
$$

where $B_{i}=\alpha_{i}+\beta_{i}-\alpha_{i} \beta_{i}$. 
The average service time of these incoming requests is

$$
\begin{aligned}
& \frac{\alpha_{i}^{\theta} c_{i}^{1-\theta} E \cdot \frac{1}{s_{i} \mu}+\left(\alpha_{i}-\alpha_{i}^{\theta} c_{i}^{1-\theta} E\right) \cdot \frac{2}{s_{i} \mu}+\left(1-\alpha_{i}\right) \beta_{i} \cdot \frac{2}{s_{i} \mu}}{\alpha_{i}^{\theta} c_{i}^{1-\theta} E+\left(\alpha_{i}-\alpha_{i}^{\theta} c_{i}^{1-\theta} E\right)+\left(1-\alpha_{i}\right) \beta_{i}} \\
= & \frac{2 \alpha_{i}+2 \beta_{i}-2 \alpha_{i} \beta_{i}-\alpha_{i}^{\theta} c_{i}^{1-\theta} E}{\alpha_{i}+\beta_{i}-\alpha_{i} \beta_{i}} \cdot \frac{1}{s_{i} \mu}=\frac{A_{i}}{B_{i}} \cdot \frac{1}{s_{i} \mu},
\end{aligned}
$$

which leads to a service rate of

$$
\mu_{i}=\frac{B_{i}}{A_{i}} s_{i} \mu
$$

where $A_{i}=2 \alpha_{i}+2 \beta_{i}-2 \alpha_{i} \beta_{i}-\alpha_{i}^{\theta} c_{i}^{1-\theta} E$.

The mean-square service time of these incoming requests is

$$
\begin{aligned}
J_{i} & =\frac{\alpha_{i}^{\theta} c_{i}^{1-\theta} E \cdot \frac{J}{s_{i}^{2}}+\left(\alpha_{i}-\alpha_{i}^{\theta} c_{i}^{1-\theta} E\right) \cdot \frac{4 J}{s_{i}^{2}}+\left(1-\alpha_{i}\right) \beta_{i} \cdot \frac{4 J}{s_{i}^{2}}}{\alpha_{i}^{\theta} c_{i}^{1-\theta} E+\left(\alpha_{i}-\alpha_{i}^{\theta} c_{i}^{1-\theta} E\right)+\left(1-\alpha_{i}\right) \beta_{i}} \\
& =\frac{4 \alpha_{i}+4 \beta_{i}-4 \alpha_{i} \beta_{i}-3 \alpha_{i}^{\theta} c_{i}^{1-\theta} E}{\alpha_{i}+\beta_{i}-\alpha_{i} \beta_{i}} \cdot \frac{J}{s_{i}^{2}}=\frac{2 A_{i}-\alpha_{i}^{\theta} c_{i}^{1-\theta} E}{B_{i}} \cdot \frac{J}{s_{i}^{2}} .
\end{aligned}
$$

From $M / G / 1$ queueing results [20], the average queueing delay at proxy $P_{i}$ is

$$
\begin{aligned}
Q_{i} & =\frac{\lambda_{i} J_{i}}{2\left(1-\frac{\lambda_{i}}{\mu_{i}}\right)}=\frac{\mu_{i} \lambda_{i} J_{i}}{2\left(\mu_{i}-\lambda_{i}\right)} \\
& =\frac{B_{i}}{A_{i}} s_{i} \mu \cdot B_{i} \lambda \cdot \frac{2 A_{i}-\alpha_{i}^{\theta} c_{i}^{1-\theta} E}{B_{i}} \cdot \frac{J}{s_{i}^{2}} \cdot \frac{1}{2\left(\frac{B_{i}}{A_{i}} s_{i} \mu-B_{i} \lambda\right)} \\
& =\frac{\left(2 A_{i}-\alpha_{i}^{\theta} c_{i}^{1-\theta} E\right) \mu \lambda J}{2 s_{i}\left(s_{i} \mu-A_{i} \lambda\right)} .
\end{aligned}
$$

We now compute the average response time of original requests. Recall that a fraction $\alpha_{i} \beta_{j}$ of the original requests go through the configured proxy $P_{j}$ and the hashed proxy $P_{i}$. If $P_{i}$ is the same as $P_{j}$, no inter-proxy communication is required. If the request results in a cache hit at $P_{i}$, the expected response time is

$$
Q_{i}+\frac{1}{s_{i} \mu}
$$

If the request causes a cache miss, $P_{i}$ issues a remote request and fetches the object from the origin server. In this case, the expected response time is

$$
Q_{i}+\frac{2}{s_{i} \mu}+L .
$$

Therefore, the average response time of the requests where $P_{i}=P_{j}$ is given by

$$
\begin{aligned}
& H\left(P_{i}\right) \cdot\left(Q_{i}+\frac{1}{s_{i} \mu}\right)+\left(1-H\left(P_{i}\right)\right)\left(Q_{i}+\frac{2}{s_{i} \mu}+L\right) \\
= & \frac{c_{i}^{1-\theta}}{\alpha_{i}^{1-\theta}} E \cdot\left(Q_{i}+\frac{1}{s_{i} \mu}\right)+\left(1-\frac{c_{i}^{1-\theta}}{\alpha_{i}^{1-\theta}} E\right)\left(Q_{i}+\frac{2}{s_{i} \mu}+L\right) .
\end{aligned}
$$


If $P_{i}$ and $P_{j}$ are different, a mean network delay $l$ is introduced due to inter-proxy communication. If the request leads to a cache hit at $P_{i}$, the expected response time is

$$
Q_{j}+\frac{2}{s_{j} \mu}+l+Q_{i}+\frac{1}{s_{i} \mu} .
$$

Otherwise, a remote request by $P_{i}$ to fetch the object from the origin server is required. In this case, the expected response time is

$$
Q_{j}+\frac{2}{s_{j} \mu}+l+Q_{i}+\frac{2}{s_{i} \mu}+L .
$$

Therefore, the average response time of the requests where $P_{i} \neq P_{j}$ is given by

$$
\begin{aligned}
& H\left(P_{i}\right) \cdot\left(Q_{j}+\frac{2}{s_{j} \mu}+l+Q_{i}+\frac{1}{s_{i} \mu}\right)+\left(1-H\left(P_{i}\right)\right)\left(Q_{j}+\frac{2}{s_{j} \mu}+l+Q_{i}+\frac{2}{s_{i} \mu}+L\right) \\
= & \frac{c_{i}^{1-\theta}}{\alpha_{i}^{1-\theta}} E \cdot\left(Q_{j}+\frac{2}{s_{j} \mu}+l+Q_{i}+\frac{1}{s_{i} \mu}\right)+\left(1-\frac{c_{i}^{1-\theta}}{\alpha_{i}^{1-\theta}} E\right)\left(Q_{j}+\frac{2}{s_{j} \mu}+l+Q_{i}+\frac{2}{s_{i} \mu}+L\right) .
\end{aligned}
$$

Consequently, the average response time of all original requests is ${ }^{3}$

$$
\begin{aligned}
& \sum_{i=1}^{n} \alpha_{i} \beta_{i}\left(\frac{c_{i}^{1-\theta}}{\alpha_{i}^{1-\theta}} E \cdot\left(Q_{i}+\frac{1}{s_{i} \mu}\right)+\left(1-\frac{c_{i}^{1-\theta}}{\alpha_{i}^{1-\theta}} E\right)\left(Q_{i}+\frac{2}{s_{i} \mu}+L\right)\right)+ \\
& \sum_{i \neq j} \alpha_{i} \beta_{j}\left(\frac{c_{i}^{1-\theta}}{\alpha_{i}^{1-\theta}} E \cdot\left(Q_{j}+\frac{2}{s_{j} \mu}+l+Q_{i}+\frac{1}{s_{i} \mu}\right)+\left(1-\frac{c_{i}^{1-\theta}}{\alpha_{i}^{1-\theta}} E\right)\left(Q_{j}+\frac{2}{s_{j} \mu}+l+Q_{i}+\frac{2}{s_{i} \mu}+L\right)\right) \\
= & \sum_{i=1}^{n} \frac{1}{s_{i} \mu}\left(A_{i}+\frac{\left(2 A_{i}-\alpha_{i}^{\theta} c_{i}^{1-\theta} E\right) \mu^{2} \lambda J B_{i}}{2\left(s_{i} \mu-A_{i} \lambda\right)}\right)+\left(1-\sum_{i=1}^{n} \alpha_{i} \beta_{i}\right) \cdot l+\sum_{i=1}^{n} \alpha_{i}\left(1-\frac{c_{i}^{1-\theta}}{\alpha_{i}^{1-\theta}} E\right) \cdot L .
\end{aligned}
$$

The first item computes the system time (which includes the service time and the queueing time) of requests at the proxies, the second item gives the inter-proxy communication delay, and the third item represents the cache miss delay. As can be seen, the average response time depends on the object allocation strategy ( $\alpha_{i}$ 's), the original request distribution $\left(\beta_{i}\right.$ 's), the speeds of the proxies $\left(s_{i}\right.$ 's), and the cache sizes $\left(c_{i}\right.$ 's).

\section{Adaptive Hash Routing Schemes}

\subsection{Static Client Configuration}

If the configured proxies are statically designated by the clients in their browsers, it is difficult for the administrator to control the volume of original requests sent to each proxy. In this case, the arrival rates $\beta_{1} \lambda, \beta_{2} \lambda, \ldots, \beta_{n} \lambda$ at the proxies can only be measured. Therefore, the response time formula derived in Section 2.2 is taken as a function $F_{S}\left(\alpha_{1}, \alpha_{2}, \ldots, \alpha_{n}\right)$ of the object allocation strategy in the optimal hash routing problem:

\footnotetext{
${ }^{3}$ The derivation of the following formula is straightforward and is omitted due to space limitation.
} 
Definition 1 [Optimal Hash Routing Problem for Static Client Configuration]

Minimize $F_{S}\left(\alpha_{1}, \alpha_{2}, \ldots, \alpha_{n}\right)$

$$
=\sum_{i=1}^{n} \frac{1}{s_{i} \mu}\left(A_{i}+\frac{\left(2 A_{i}-\alpha_{i}^{\theta} c_{i}^{1-\theta} E\right) \mu^{2} \lambda J B_{i}}{2\left(s_{i} \mu-A_{i} \lambda\right)}\right)+\left(1-\sum_{i=1}^{n} \alpha_{i} \beta_{i}\right) \cdot l+\sum_{i=1}^{n} \alpha_{i}\left(1-\frac{c_{i}^{1-\theta}}{\alpha_{i}^{1-\theta}} E\right) \cdot L,
$$

where

$$
A_{i}=2 \alpha_{i}+2 \beta_{i}-2 \alpha_{i} \beta_{i}-\alpha_{i}^{\theta} c_{i}^{1-\theta} E
$$

and

$$
B_{i}=\alpha_{i}+\beta_{i}-\alpha_{i} \beta_{i}
$$

subject to the constraints $\sum_{i=1}^{n} \alpha_{i}=1$ and $\alpha_{i} \geq 0(i=1,2, \ldots, n)$.

This non-linear resource allocation problem can be solved using the Lagrange multiplier theorem. It is equivalent to solving the following set of equations for $\alpha_{1}, \alpha_{2}, \ldots, \alpha_{n}$, and $k$ :

$$
\left\{\begin{aligned}
\frac{\partial F_{S}}{\partial \alpha_{i}} & =k, \quad(i=1,2, \ldots, n) \\
\sum_{i=1}^{n} \alpha_{i} & =1
\end{aligned}\right.
$$

Since $\frac{\partial F_{S}}{\partial \alpha_{i}}$ is independent of $\alpha_{j}$ 's where $j \neq i$, it is easy to solve this set of equations numerically (e.g., using a bisection method [21]).

The corresponding adaptive hash routing scheme works as follows. The proxies monitor their local arrival rates of original requests and periodically adjust the object allocation strategy. The interval between two consecutive adjustments is called a sampling interval. At each adjustment point, the measured arrival rates $\beta_{1} \lambda, \beta_{2} \lambda, \ldots, \beta_{n} \lambda$ of the previous sampling interval are used to parameterize the optimal hash routing problem. The object allocation strategy is then adjusted based on the optimal solution to the problem. The optimal solution can be computed by one of the proxies (suppose it is proxy $P_{i}$ ). At each adjustment point, all proxies send their measured arrival rates to $P_{i}$. On receiving the measured rates, $P_{i}$ computes the new object allocation strategy and broadcasts it to the other proxies. Note that only the hash function specifying the object to proxy allocation changes at the time of adjustment. The objects in the caches are not physically moved between the proxies. Original requests arriving after the adjustment would be directed to the proxies based on the updated hash function. Therefore, the cache contents of the proxies would be adjusted automatically by the cache replacement algorithm. This adaptive scheme is referred to as the ADA-OBJ (Adaptive Object Allocation) scheme.

\subsection{Dynamic Client Configuration}

In dynamic client configuration, all proxies share the same logical name (e.g., proxy.site.com). Before issuing an original request, the client first sends a query to a domain name server (DNS) for name-to-address resolution. The query result returned from the DNS contains the IP address of one of the proxies and a time-to-live (TTL) 
value. The client caches the query result for a period equal to TTL. During this period, the client uses the cached IP address to direct original requests. On expiration of the TTL period, the client queries the DNS again. With dynamic client configuration, the administrator can adjust the fraction of original requests sent to each proxy by tuning the probability of mapping DNS queries to the proxy. We shall refer to these probabilities as the DNS allocation strategy, i.e., $\left(\beta_{1}, \beta_{2}, \ldots, \beta_{n}\right)$. Note that both object and DNS allocation strategies can be optimized under dynamic client configuration. Therefore, the response time formula derived in Section 2.2 is taken as a function $F_{D}\left(\alpha_{1}, \alpha_{2}, \ldots, \alpha_{n}, \beta_{1}, \beta_{2}, \ldots, \beta_{n}\right)$ of the two allocation strategies in the optimal hash routing problem:

Definition 2 [Optimal Hash Routing Problem for Dynamic Client Configuration]

Minimize $F_{D}\left(\alpha_{1}, \alpha_{2}, \ldots, \alpha_{n}, \beta_{1}, \beta_{2}, \ldots, \beta_{n}\right)$

$$
=\sum_{i=1}^{n} \frac{1}{s_{i} \mu}\left(A_{i}+\frac{\left(2 A_{i}-\alpha_{i}^{\theta} c_{i}^{1-\theta} E\right) \mu^{2} \lambda J B_{i}}{2\left(s_{i} \mu-A_{i} \lambda\right)}\right)+\left(1-\sum_{i=1}^{n} \alpha_{i} \beta_{i}\right) \cdot l+\sum_{i=1}^{n} \alpha_{i}\left(1-\frac{c_{i}^{1-\theta}}{\alpha_{i}^{1-\theta}} E\right) \cdot L,
$$

where

$$
A_{i}=2 \alpha_{i}+2 \beta_{i}-2 \alpha_{i} \beta_{i}-\alpha_{i}^{\theta} c_{i}^{1-\theta} E
$$

and

$$
B_{i}=\alpha_{i}+\beta_{i}-\alpha_{i} \beta_{i}
$$

subject to the constraints $\sum_{i=1}^{n} \alpha_{i}=1, \sum_{i=1}^{n} \beta_{i}=1, \alpha_{i} \geq 0$ and $\beta_{i} \geq 0(i=1,2, \ldots, n)$.

Similar to the static case, the above non-linear resource allocation problem can be solved using the Lagrange multiplier theorem. It is equivalent to solving the following set of equations for $\alpha_{1}, \alpha_{2}, \ldots, \alpha_{n}, \beta_{1}, \beta_{2}, \ldots, \beta_{n}, k_{1}$, and $k_{2}$ :

$$
\left\{\begin{array}{rlrl}
\frac{\partial F_{D}}{\partial \alpha_{i}} & =k_{1}, & & (i=1,2, \ldots, n) \\
\frac{\partial F_{D}}{\partial \beta_{i}} & =k_{2}, & & (i=1,2, \ldots, n) \\
\sum_{i=1}^{n} \beta_{i} & =1, \\
\sum_{i=1}^{n} \alpha_{i} & =1 .
\end{array}\right.
$$

Since both $\frac{\partial F_{D}}{\partial \alpha_{i}}$ and $\frac{\partial F_{D}}{\partial \beta_{i}}$ are independent of $\alpha_{j}$ 's and $\beta_{j}$ 's where $j \neq i$, this set of equations can be solved numerically (e.g., using a bisection method [21]).

The corresponding adaptive hash routing scheme consists of monitoring $\lambda$, the aggregate arrival rate of original requests, and periodically adjusting the object and DNS allocation strategies. At each adjustment point, the measured arrival rate $\lambda$ of the previous sampling interval is used to parameterize the optimal hash routing problem. The domain name server is then instructed to map DNS queries to the proxies according to the DNS allocation strategy in the optimal solution. This can be done by using a weighted round-robin algorithm [14] in DNS scheduling. The web objects, on the other hand, are hashed to the proxies based on the object allocation strategy in the optimal solution. Similar to ADA-OBJ, the objects in the caches are not physically moved between 
the proxies at the time of adjustment. This adaptive scheme is referred to as the ADA-OBJ/DNS (Adaptive Object and DNS Allocations) scheme. Since more variables $\left(\alpha_{i}^{\prime} s\right.$ and $\left.\beta_{i}^{\prime} s\right)$ are available for adjustment under dynamic client configuration compared to static configuration, the ADA-OBJ/DNS scheme is expected to outperform the ADA-OBJ scheme.

\section{Experimental Configurations}

A trace-driven simulator was developed to evaluate hash routing performance. The traces, simulation model, cache replacement algorithm, and hash function used are described in this section.

\subsection{Traces}

Our experimental study made use of client-side web proxy traces from different sources (DEC [22] and Boeing [23]) to simulate the requests made by clients. Similar performance trends were observed for these two sets of traces and indeed for different daily traces. Due to space limitation, we shall report the results using the traces collected on a Boeing web proxy between 3-1-1999 and 3-5-1999 (five traces, one per day) in this paper. ${ }^{4}$ Each trace entry contains the request time, the client ID, the target URL, the size of the target object, and other information about the requests. The number of entries in each daily trace is over 4 million. The requests are generated from more than 30000 clients. The average size of the web objects accessed is about $12.5 \mathrm{~KB}$. The total size of all distinct objects in each trace is around 17 GB.

\subsection{Simulation Model}

We simulated 8 web proxies with an aggregate processing speed of 8.0. Each proxy consists of a CPU server and a cache manager. The CPU server processes requests in First-Come-First-Serve (FCFS) order. In general, a reply message cannot be processed until the requested object is obtained from the origin server or the hashed proxy. Therefore, processing a request and the associated reply is considered to be two separate tasks in our simulation. The two tasks are processed consecutively by the same proxy only if the request leads to a cache hit at that proxy. To achieve the default CPU utilization of 75\% during the peak hours (between 10:00 and 13:00) of the day, the normalized CPU time (i.e., the CPU time on a baseline proxy) for processing a request message was set at $260 \mu \mathrm{s}$. The normalized CPU time $t$ for processing a reply message is assumed to grow linearly with size $s$ of the requested object: $t=t_{a}+t_{b} \cdot s$, where $t_{a}$ and $t_{b}$ were set at $260 \mu s$ and $48 \mu s / \mathrm{KB}$ respectively. The ratio of $t_{a}$ to $t_{b}$ was chosen based on data reported in the literature [24]. Under this setting, the normalized mean and mean-square processing times of a request and the associated reply were $0.87 \mathrm{~ms}$ and $52 \mathrm{~ms}$-square respectively for the traces we used. To simulate different levels of workloads, we changed the time scale of

\footnotetext{
${ }^{4}$ To the best of our knowledge, the Boeing traces are the most recent client-side proxy traces publically available.
} 
the traces accordingly. The network delay due to inter-proxy communication was set proportional to the size of request/response messages and had an average value of 0.2 seconds. The average cache miss delay of fetching an object from the origin server was assumed to follow an exponential distribution with a mean value of 4.0 seconds.

As mentioned in Section 3, the adaptive hash routing schemes periodically adjust the object and DNS allocations based on the traffic pattern. At each adjustment point, they make use of the statistics collected in the previous sampling interval to parameterize the optimal hash routing problem. The computed allocation strategy is then applied to the cluster of proxies in our trace-driven simulation experiments. The default length of the sampling interval was set at 30 seconds. The impact of sampling interval on performance is investigated and presented in Section 5.3. Since there was no previous work on optimizing object and DNS allocations for hash routing, we compared our proposed schemes with a static speed-based hash routing scheme called SPEED. SPEED is an intuitively attractive scheme that allocates the fractions of web objects to the proxies proportionally to proxy speeds, i.e., $\alpha_{i}=s_{i} / \sum_{i=1}^{n} s_{i}$. Faster proxies are allocated more objects. The object allocation strategy of SPEED is static and does not change with time.

For static client configuration, the client to proxy association was constructed based on speed-proportional or zipf-like distributions. For dynamic client configuration, the default TTL value was set at 15 seconds. The impact of TTL duration is examined and discussed in Section 5.5. Each simulation run started with all caches being empty. The first one third of the trace was considered the start up period and was run to bring the caches into a relatively steady state. Statistics were collected for the remaining two thirds of the trace only.

\subsection{Cache Replacement Algorithm}

A generalized LFU replacement algorithm (GLFU) similar to the one proposed in [2] was implemented for each cache manager. The algorithm incorporates access frequencies and sizes of web objects to optimize replacement decisions. For each object $O$, we refer to the product $f(O) \cdot s(O)$ as the generalized size (GSize) of the object, where $f(O)$ and $s(O)$ are the access frequency and size of object $O$ respectively. The GSize value reflects the benefit of caching an object, i.e., the traffic reduction between the proxy and the origin server. To accommodate a new object, one or more objects may need to be removed from the cache. In order to maximize the overall caching benefit, the replacement candidates should be selected such that they involve the least total benefit while creating enough space. This is equivalent to the knapsack problem which is NP-complete [16]. A fast greedy heuristic is to arrange the objects in the cache in increasing order of access frequency. When an object $P$ not in the cache is requested, the cached objects are examined and marked sequentially until sufficient space for $P$ is created. The total GSize of the marked objects is then compared with the GSize of $P$. If the GSize of $P$ is larger, the marked objects are removed and $P$ is inserted into the cache. Otherwise, the cache contents are not updated since the benefit of caching $P$ is outweighed by the performance loss that would result from removing the marked objects. This checking process is referred to as object admission control [2]. The access frequency of an object 
is dynamically estimated using a "sliding window" technique [3] covering the $K$ most recent references of the object, i.e., $f(O)=\frac{K}{t-t_{K}}$, where $t$ is the current time and $t_{K}$ is the $K$ th most recently referenced time. $K$ was set at 3 in our experiments. For the purpose of GSize evaluation, the reference times of objects not in the cache must be maintained. They are stored in the form of object descriptors in an auxiliary cache at each proxy whose size is negligible compared to the main cache that stores the actual objects. Interested readers are referred to [2] and [5] for detailed description of the auxiliary cache. Simple LFU replacement algorithm is used to manage the object descriptors. In our simulation experiments, the proxies had equal size caches. The aggregate cache size of all proxies was set at $10 \%$ of the total size of all distinct objects accessed in each trace. The size of each auxiliary cache (described in terms of the number of object descriptors) was set to twice the number of objects the main cache can hold.

\subsection{Hash Function}

A hash function called HRW (Highest Random Weight) was used to map the URLs to the proxies in our experiments. This function was first proposed by Thaler et al. [15] and was extended by Ross [7] to handle non-uniform object allocation strategy. HRW tries to minimize the fraction of objects that need to be remapped to a different proxy when the object allocation strategy changes.

HRW first maps web objects to an $n$-dimension hash space, where $n$ is the number of proxies. The value of the $i$ th dimension is given by [15]:

$$
\begin{aligned}
W\left(U R L, P R O X Y_{i}\right)= & \left(1 1 0 3 5 1 5 2 4 5 \cdot \left(\left(1103515245 \cdot A\left(P R O X Y_{i}\right)+12345\right)\right.\right. \\
& X O R D(U R L))+12345)\left(\bmod 2^{31}\right) / 2^{31},
\end{aligned}
$$

where $A\left(P R O X Y_{i}\right)$ is the address of the proxy $i$ and $D(U R L)$ is a 31-bit digest of the URL. In our simulation, $D(U R L)$ is taken to be the unique URL ID provided in the Boeing traces [23]. The hash space is partitioned among the proxies by a set of multipliers $x_{1}, x_{2}, \ldots, x_{n}$. An $n$-dimension vector $\left(v_{1}, v_{2}, \ldots, v_{n}\right)$ is assigned to proxy $i$ if $v_{i} \cdot x_{i}=\max \left\{v_{j} \cdot x_{j}, j=1,2, \ldots, n\right\}$. The multipliers are set based on the object allocation strategy $\left(\alpha_{1}, \alpha_{2}, \ldots, \alpha_{n}\right)$ (without loss of generality, assume $\left.\alpha_{1} \leq \alpha_{2} \leq \cdots \leq \alpha_{n}\right)$ [7]:

$$
\left\{\begin{array}{l}
x_{1}=\left(n \alpha_{1}\right)^{\frac{1}{n}} \\
x_{i}=\left(\frac{(n-i+1) \cdot\left(\alpha_{i}-\alpha_{i-1}\right)}{\prod_{j=1}^{i-1} x_{j}}+x_{i-1}^{n-i+1}\right)^{\frac{1}{n-i+1}} \cdot \quad(i=2,3, \ldots, n)
\end{array}\right.
$$

\section{Performance Results}

\subsection{Zipf-like Distribution}

First, we analyze the Zipf-like distribution of web accesses in the traces and determine the values of $\theta$ and $E$ to be used in our simulation experiments. Consider a single proxy cache. From Section 2.2, if the requests to 
objects follow a Zipf-like distribution with parameter $\theta$, the cache hit ratio $H$ can be calculated as follows:

$$
H=c^{1-\theta} \cdot E,
$$

where $c$ is the cache size described in the unit of mean object size (about $12.5 \mathrm{~KB}$ ) and $E$ is a constant. $c$ is called the normalized cache size. It is easy to see that $H$ grows linearly with $c$ on the log scale:

$$
\log H=(1-\theta) \cdot \log c+\log E .
$$

We simulated one proxy with cache sizes from 7000 to 140000 units (corresponding to a range from $0.5 \%$ to $10 \%$ of the total size of all distinct objects accessed). Figure 3 shows cache hit ratio $H$ as a function of cache size $c$ for the 3-1-1999 trace. As can be seen, the experimental points fit a straight line quite well. The plots for the other traces are similar and are not shown due to space limitation.

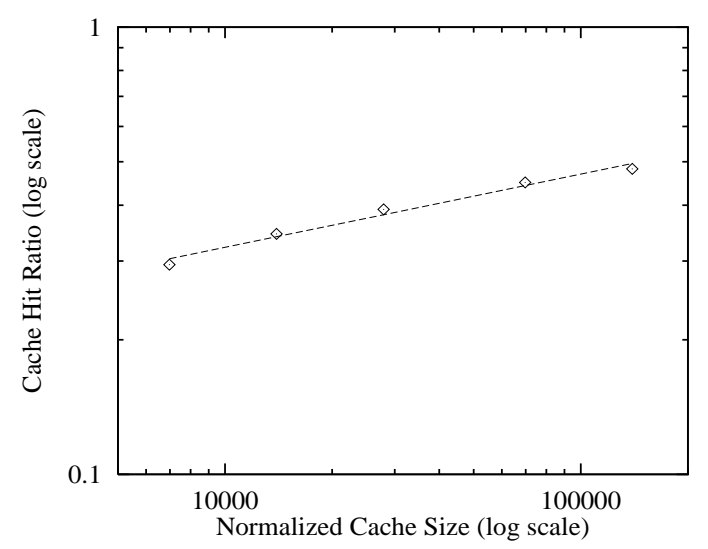

Figure 3: Cache Hit Ratios of Different Cache Sizes for the 3-1-1999 trace

We computed the parameters $\theta$ and $\log E$ using the least square method for each trace. As shown in Table 2, $\theta$ and $\log E$ remain fairly stable over different days. In subsequent experiments, $\theta$ and $E$ are set to 0.843 and 0.074 respectively (i.e., $\log E=-2.6$ ).

Table 2: $\theta$ and $\log E$ for Different Traces

\begin{tabular}{|c|c|c|}
\hline Trace & $\theta$ & $\log E$ \\
\hline $3-1-1999$ & 0.836 & -2.643 \\
\hline $3-2-1999$ & 0.837 & -2.653 \\
\hline $3-3-1999$ & 0.851 & -2.408 \\
\hline $3-4-1999$ & 0.848 & -2.477 \\
\hline $3-5-1999$ & 0.845 & -2.544 \\
\hline
\end{tabular}


Next, we evaluated the hit ratio prediction formula used in Section 2.2:

$$
H\left(P_{i}\right)=\frac{c_{i}^{1-\theta}}{\alpha_{i}^{1-\theta}} \cdot E
$$

In the first set of experiments, the 8 proxies were given equal size caches (17500 units), but were allocated different fractions of objects in hash routing. The object allocation strategy was set to $(0.267,0.267,0.133$, $0.133,0.067,0.067,0.033,0.033)$. Table 3 shows the predicted and measured cache hit ratios of each proxy for the 3-1-1999 trace. It is intuitive that those proxies that are associated with more objects, i.e., allocated higher fractions of objects, will have a lower chance of cache hit. This is shown to be the case as the predicted and measured cache hit ratios match reasonably well.

Table 3: Comparison of Predicted and Measured Cache Hit Ratios (Set 1)

\begin{tabular}{|c|c|c|c|c|c|c|c|c|}
\hline Proxy ID & 1 & 2 & 3 & 4 & 5 & 6 & 7 & 8 \\
\hline Fraction of Objects & 0.267 & 0.267 & 0.133 & 0.133 & 0.067 & 0.067 & 0.033 & 0.033 \\
\hline Predicted Hit Ratio & 0.424 & 0.424 & 0.472 & 0.472 & 0.526 & 0.526 & 0.588 & 0.588 \\
\hline Measured Hit Ratio & 0.439 & 0.440 & 0.462 & 0.479 & 0.522 & 0.491 & 0.521 & 0.579 \\
\hline
\end{tabular}

In the second set of experiments, the 8 proxies were allocated equal fractions of objects in hash routing, but had different size caches (1750, 1750, 5250, 5250, 15750, 15750, 47250 and 47250 units). Table 4 shows the results for the 3-1-1999 trace. As expected, the larger the cache size, the higher the cache hit ratio. Again, the measured values agree with the predicted ones quite well.

Table 4: Comparison of Predicted and Measured Cache Hit Ratios (Set 2)

\begin{tabular}{|c|c|c|c|c|c|c|c|c|}
\hline Proxy ID & 1 & 2 & 3 & 4 & 5 & 6 & 7 & 8 \\
\hline Normalized Cache Size & 1750 & 1750 & 5250 & 5250 & 15750 & 15750 & 47250 & 47250 \\
\hline Predicted Hit Ratio & 0.332 & 0.332 & 0.395 & 0.395 & 0.469 & 0.469 & 0.558 & 0.558 \\
\hline Measured Hit Ratio & 0.329 & 0.330 & 0.409 & 0.425 & 0.473 & 0.478 & 0.538 & 0.511 \\
\hline
\end{tabular}

\subsection{Effect of Load Balancing}

In this section, we examine the impact of adaptive hash routing on load distribution under static client configuration. The 8 proxies were assigned equal speed and each proxy received original requests from 1/8 of the clients. Figures 4(a) and (b) show the average response times of the entire trace and during the peak hours respectively for different days. 


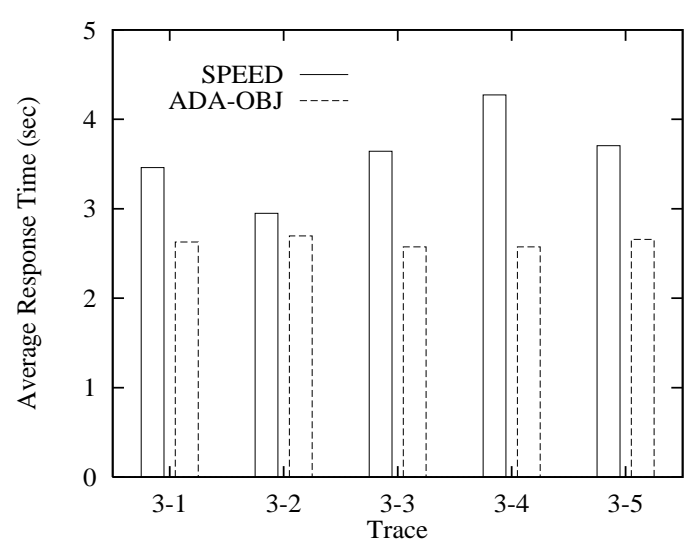

(a) Results of Entire Trace

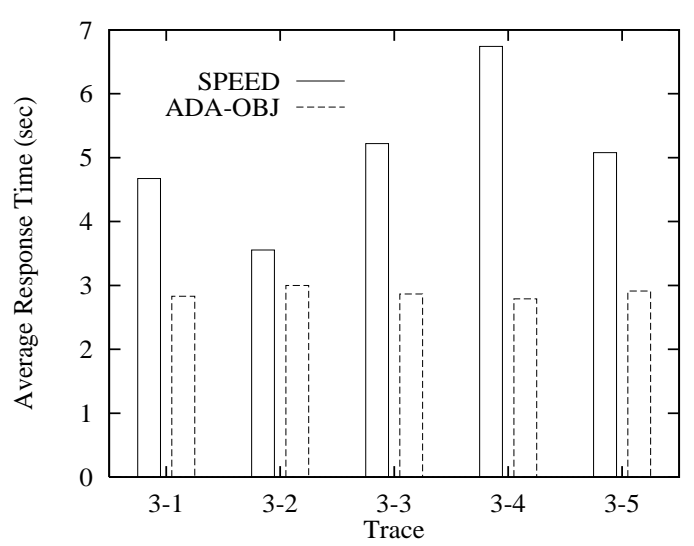

(b) Results during Peak Hours only

Figure 4: Average Response Time for Different Days

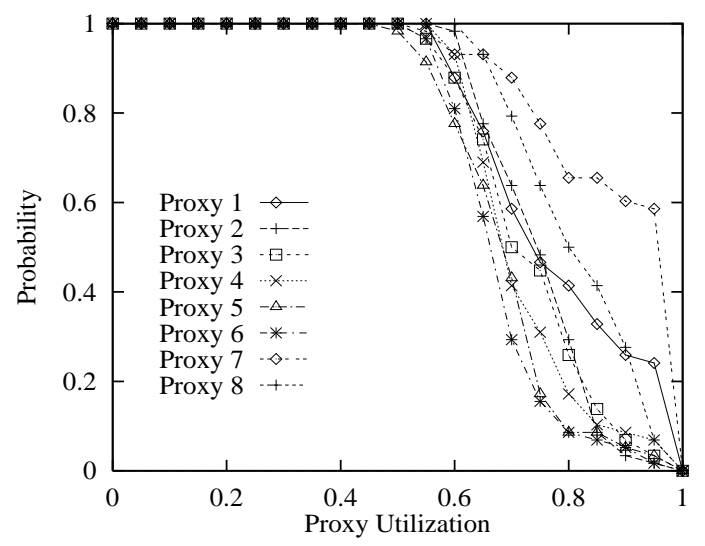

(a) SPEED

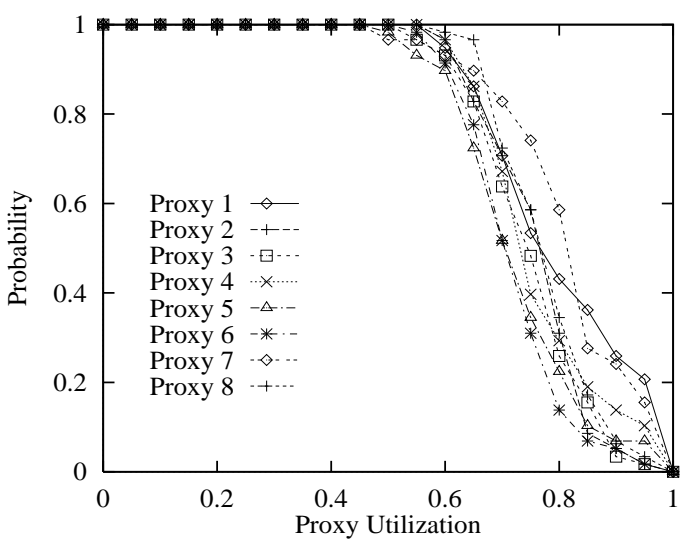

(b) ADA-OBJ

Figure 5: Distribution of Proxy Utilization for the 3-1-1999 Trace

As can be seen, ADA-OBJ reduces average response time compared to SPEED, and the improvement is more significant during the peak hours when the workload is heavier. This is because ADA-OBJ achieves better load balance by dynamically adjusting the object allocation. Figure 5(a) shows the distribution of proxy utilization for SPEED during the peak hours of 3-1-1999. Each data point $(x, y)$ shows the probability of proxy utilization being higher than $x$ is $y$. A proxy is more heavily loaded if its curve is closer to the upper-right corner, and is less heavily loaded if its curve is closer to the lower-left corner. It is seen from Figure 5(a) that even when the client to proxy association and the object allocation are both evenly distributed under SPEED, the load distribution among the proxies can be quite unbalanced. Proxies 7 and 8 are more than $85 \%$ utilized for $66 \%$ and $41 \%$ of the time respectively, while proxies 5 and 6 are more than $85 \%$ utilized for only $9 \%$ and $7 \%$ of the time respectively. In contrast, as shown in Figure 5(b), the proxy loads are much more balanced under ADA-OBJ. The probability of the utilization being higher than $85 \%$ varies in a much narrower range (7\% to $36 \%$ ) for different proxies. 
Other than network delay and machine speeds, the mean response time perceived by the clients depends on both the average queueing delay and cache miss delay of the requests. Figures 4 and 5 have shown that by optimizing object allocation, ADA-OBJ significantly reduces the load imbalance among the proxies and hence reduces the queueing delay. One tradeoff of ADA-OBJ is that the fractions of web objects allocated to different proxies may be different. This can potentially decrease the number of cache hits because all proxies had equal size caches in our experiments. Moreover, the number of cache misses may increase due to periodic adjustment of object allocation. We evaluated the impact of adaptive hash routing on cache hit ratio. Figure 6 shows that the cache hit ratio of ADA-OBJ is only marginally lower (about 1\%) than that of SPEED for all the traces tested. The result has taken into consideration all the adjustments of object allocation made in the simulation. It implies adaptive hash routing introduces little penalty on cache miss delay. Since the reduction in queueing delay substantially outweighs the increase in cache miss delay, ADA-OBJ greatly improves the average response time of the requests (see Figure 4).

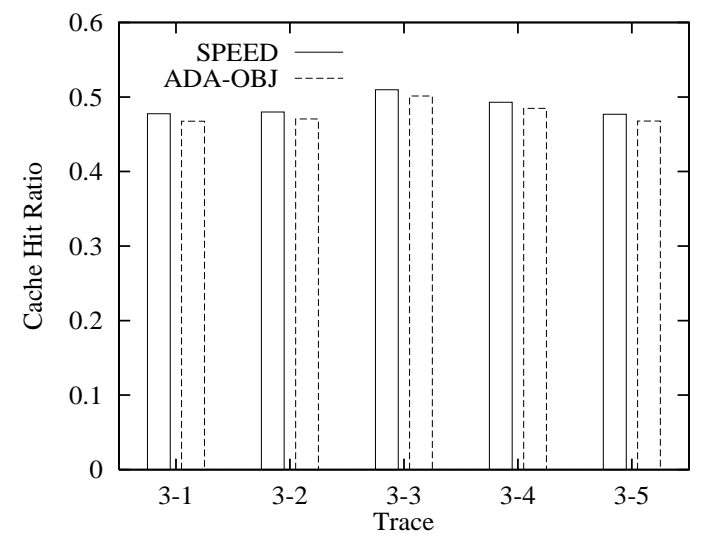

Figure 6: Cache Hit Ratio for Different Days

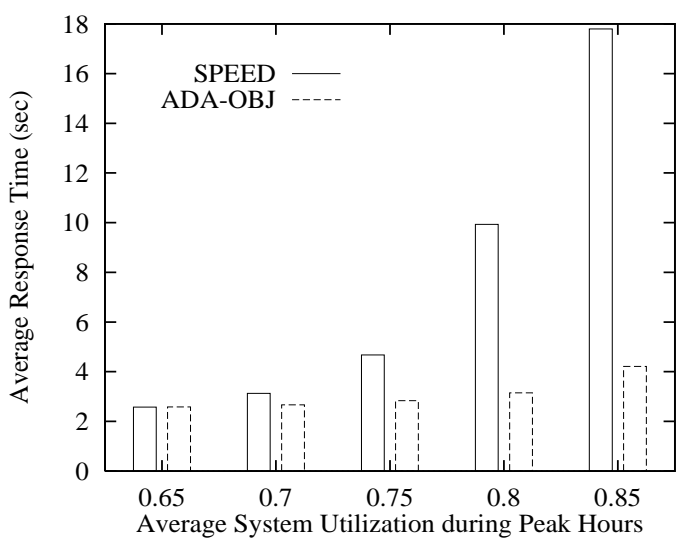

Figure 7: Average Response Time for Different System Utilizations

To see the impact of system load, we changed the time scale of the traces by different factors. Figure 7 shows 
the response time during the peak hours of 3-1-1999 as a function of average system utilization. ${ }^{5}$ It is clearly seen that the higher the system utilization, the more substantial the benefit of load balancing. For example, ADA-OBJ outperforms SPEED by $76 \%$ at an average utilization of $80 \%$, in which case the queueing delay dominates the response time. On the other hand, when the system is lightly loaded, ADA-OBJ and SPEED produce similar response times as expected.

\subsection{Impact of Adjustment Frequency}

To investigate the impact of adjustment frequency, we conducted simulations of ADA-OBJ for different sampling intervals. The shorter the sampling interval, the higher the adjustment frequency. Figure 8 shows the performance for the 3-1-1999 trace when the average system utilization during the peak hours was set at $75 \%$. The results of SPEED are also shown in the figure for comparison purposes.

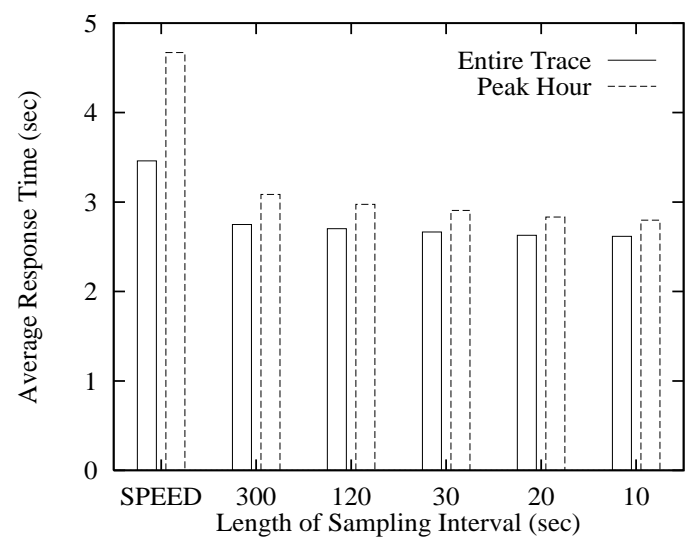

(a) Average Response Time

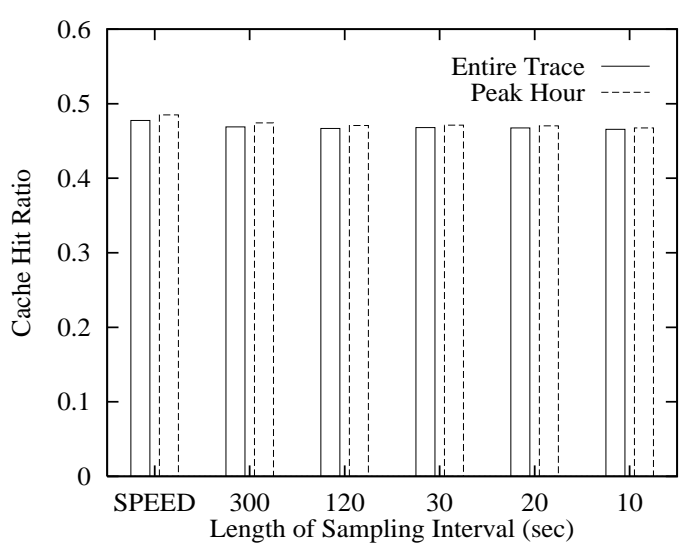

(b) Cache Hit Ratio

Figure 8: Performance for Different Adjustment Frequencies

In general, the average response time decreases with increasing adjustment frequency, i.e., when ADA-OBJ is more adaptive to the workload (see Figure 8(a)). However, the performance difference is not significant among the sampling intervals tested. An adjustment frequency of once every 5 minutes is able to achieve considerable improvement with respect to SPEED. Meanwhile, the higher the adjustment frequency, the larger the number of objects reallocated by ADA-OBJ. Figure 8(b) shows the cache hit ratio as a function of the adjustment frequency. It can be seen that the cache hit ratio decreases very little as the sampling interval gets shorter. This further demonstrates that the adjustment of object allocation has negligible impact on caching performance.

\footnotetext{
${ }^{5}$ The results of different daily traces exhibit similar performance trends in this and subsequent sets of experiments. Therefore, only the results of one trace (the 3-1-1999 trace) are reported in the rest of this paper.
} 


\subsection{Impact of Skewed Client to Proxy Association}

Client to proxy association was assumed to be uniformly distributed in the experiments reported so far. To study the impact of skewed client association, ${ }^{6}$ we associated the clients with the proxies based on a Zipflike distribution. Specifically, a fraction $i^{-\sigma} / r$ of the clients were associated with proxy $i$, where $\sigma \geq 0$ and $r=\sum_{i=1}^{8} i^{-\sigma}$ is a normalization factor. Figure 9 shows the client distributions among the 8 proxies for different $\sigma$ 's. The larger the value of $\sigma$, the more skewed the client distribution.

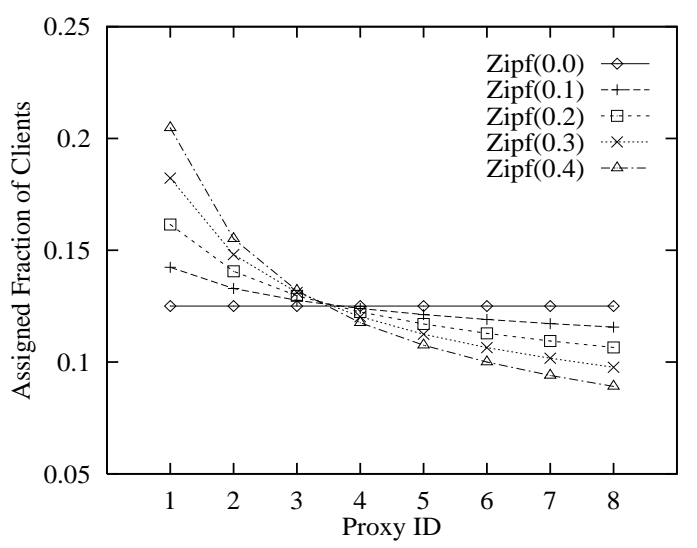

Figure 9: Client Distribution for Different Zipf Parameters

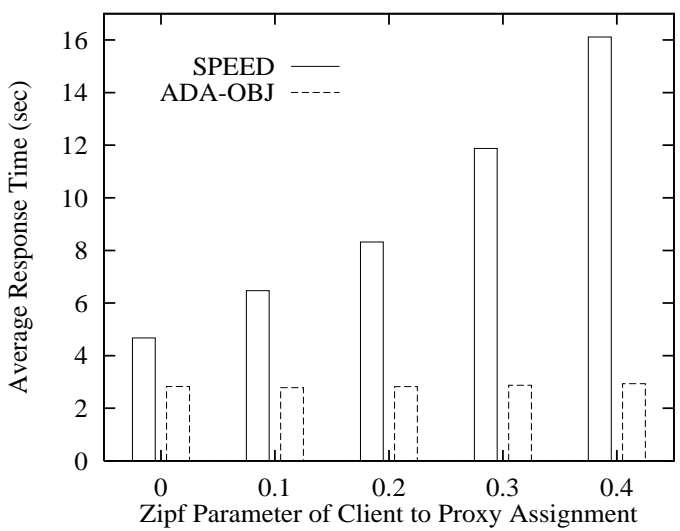

Figure 10: Average Response Time for Different Client Distributions

Figure 10 shows the average response time during the peak hours of the 3-1-1999 trace for different Zipf-like client associations. It can be observed that the performance of SPEED deteriorates substantially and rapidly with increasing skewness in client association. This is because SPEED allocates the same proportion of objects to each proxy regardless of the client association. A larger set of clients normally implies a higher aggregate rate of original requests. Therefore, the proxies experience highly uneven loadings. In contrast, ADA-OBJ dynamically

\footnotetext{
${ }^{6}$ Note that the administrator does not have control of the association in static client configuration.
} 
estimates the rate of original requests sent to each proxy and adjusts object allocation accordingly. As a result, higher fractions of objects are allocated to proxies with lower volume of clients associated, thereby achieving more even workload distribution. It can be seen from Figure 10 that skewed client association hardly affects the performance of ADA-OBJ (dashed bars). This suggests the negative impact of skewed client association can be greatly alleviated by properly adjusting the object allocation strategy.

\subsection{Performance for Dynamic Client Configuration and Impact of Heterogeneous Proxy Speeds}

So far, we have been evaluating hash routing performance under static client configuration. In this section, we compare ADA-OBJ/DNS with SPEED and ADA-OBJ under dynamic client configuration and study the impact of heterogeneous proxy speeds. In our experiments, the 8 proxies were divided into two groups: 4 fast proxies and 4 slow proxies. The aggregate proxy speed was fixed at 8.0 and the speed ratio between the fast and slow proxies was varied from 1 to 5 . The average system utilization during the peak hours was set at $75 \%$. It is known that dynamic client configuration can reduce the skewness of original request distribution [25]. To allow for fair comparisons, the client requests were also routed through a domain name server in the simulation of SPEED and ADA-OBJ. They are referred to as SPEED* and ADA-OBJ* respectively to distinguish the results from those in the previous sections. The probability of mapping DNS queries to a proxy was set to be proportional to the proxy's speed in these two schemes while that in ADA-OBJ/DNS was set based on the optimal solution to the resource allocation problem presented in Section 3.2. Figure 11 shows the average response time during the peak hours of the 3-1-1999 trace.

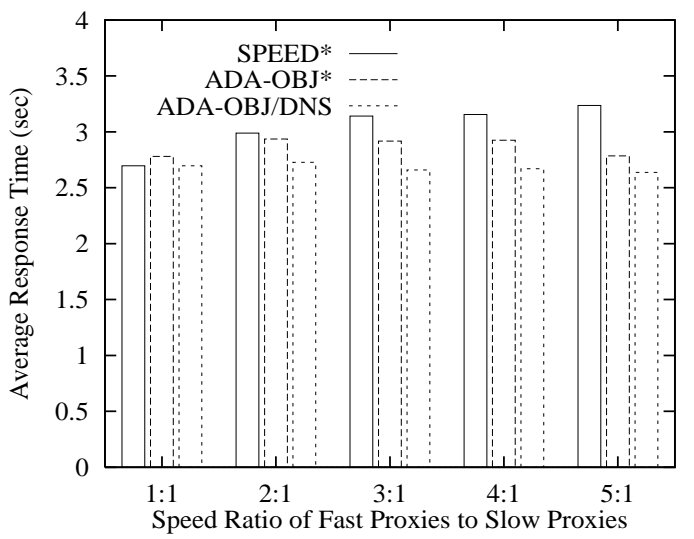

Figure 11: Average Response Time for Proxies of Different Speeds

It is observed from Figure 11 that ADA-OBJ* and ADA-OBJ/DNS outperform SPEED* when the proxies have different speeds. This is due to the different workload distributions among the proxies by the three schemes. From the analytical model in Section 2, it is easy to see that the proxy utilization $\frac{\lambda_{i}}{\mu_{i}}=\frac{\left(2 \alpha_{i}+2 \beta_{i}-2 \alpha_{i} \beta_{i}-\alpha_{i}^{\theta} c_{i}^{1-\theta} E\right) \lambda}{s_{i} \mu}$ is not a linear function of $\alpha_{i}$ and $\beta_{i}$. Therefore, the fast and slow proxies are not equally utilized when original 


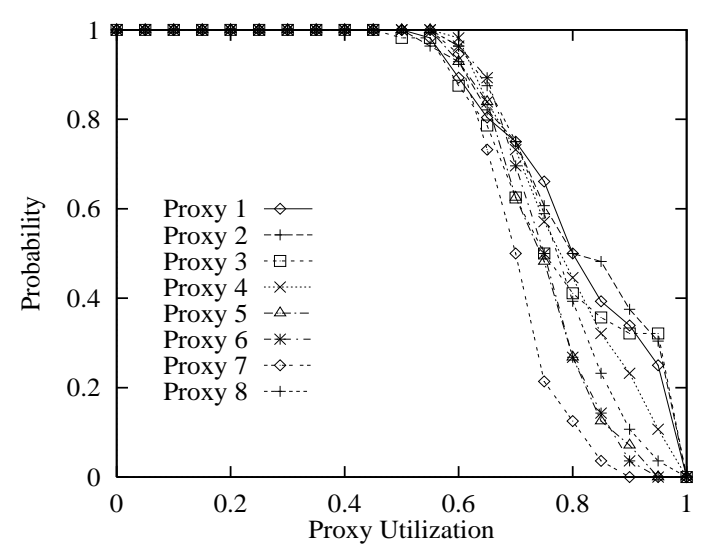

(a) SPEED*

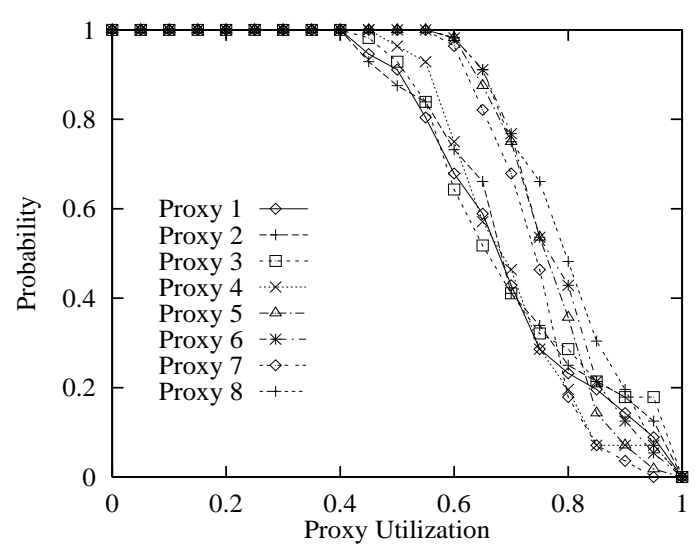

(b) ADA-OBJ*

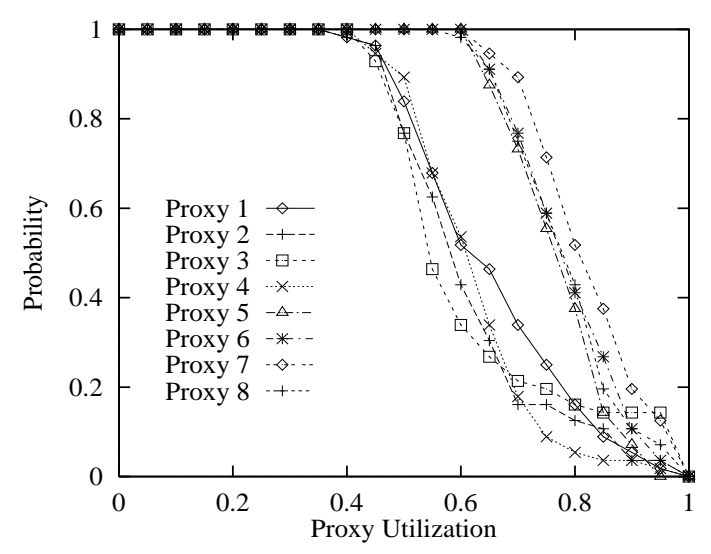

(c) ADA-OBJ/DNS

Figure 12: Distribution of Proxy Utilization for Speed Ratio of 4

requests and web objects are both proportionally distributed to the proxies based on their speeds. Figure 12(a) shows the distribution of proxy utilization for SPEED* at the speed ratio of 4 . As can be seen, the slow proxies ( 1 , 2, 3 and 4) are much more heavily loaded than the fast proxies (5, 6, 7 and 8). In contrast, our proposed schemes (ADA-OBJ* and ADA-OBJ/DNS) move some workload from the slow proxies to the fast proxies by assigning more objects and/or mapping more DNS queries to the fast proxies. This is clearly shown in Figures 13(a) and (b), which plot an example of the fractions of web objects and DNS mappings respectively assigned to each proxy by different schemes. Consequently, as shown in Figures 12(b) and (c), ADA-OBJ* and ADA-OBJ/DNS significantly reduce the utilization of the slow proxies but do not increase that of the fast proxies much due to their higher speeds. Hence, substantial performance improvement over SPEED is achieved. Moreover, note that the fast proxies are more heavily loaded than the slow proxies under the optimized schemes ADA-OBJ* and ADAOBJ/DNS (see Figures 12(b) and (c)). This is consistent with job scheduling results in distributed systems [14]. The results shown in Figures $11-13$ imply that hash routing performance can be greatly enhanced by properly 
adjusting the object and DNS allocations when the proxies have different speeds. ${ }^{7}$

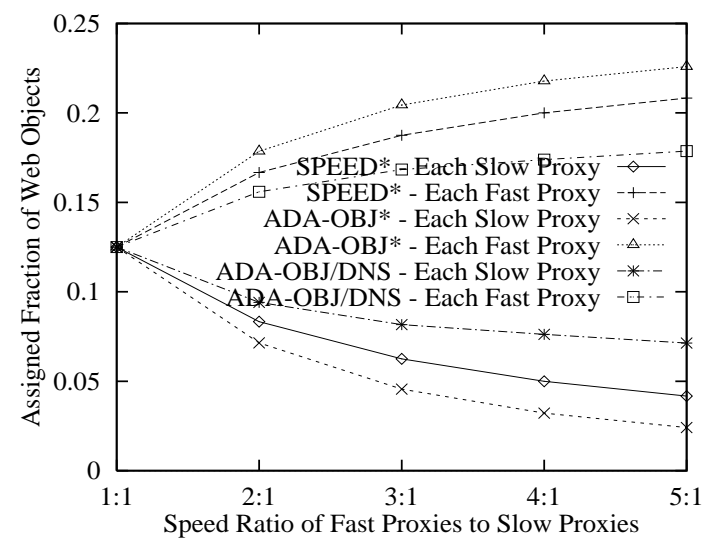

(a) Object Allocation Strategy

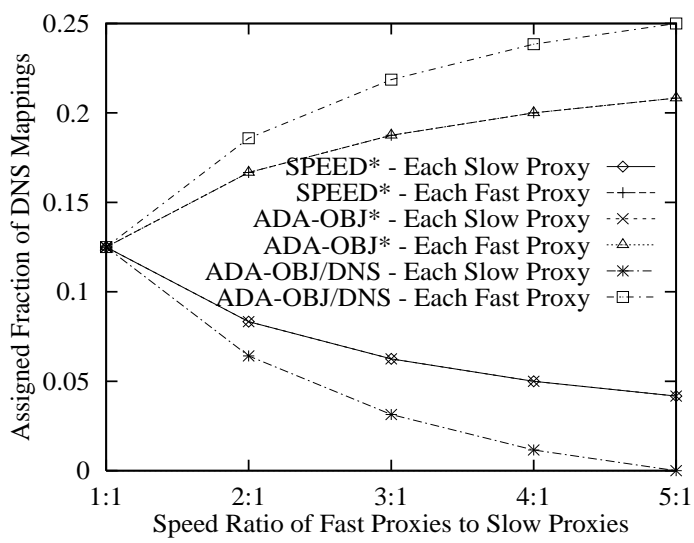

(b) DNS Allocation Strategy

Figure 13: Object and DNS Allocation Strategies for Proxies of Different Speeds

From Figures 13(a) and (b), it is also seen that ADA-OBJ/DNS and ADA-OBJ* use different methods to regulate the workload distribution. ADA-OBJ/DNS adjusts both object and DNS allocations while ADA-OBJ* adjusts the object allocation only. Thus, the object allocation strategy of ADA-OBJ/DNS is less skewed than that of ADA-OBJ*. As a result, ADA-OBJ/DNS obtains a slightly higher cache hit ratio than ADA-OBJ* (see Figure 14). The hit ratios of ADA-OBJ/DNS and SPEED are hardly distinguishable. This further demonstrates adaptive hash routing has little penalty on the cache miss delay compared to static hash routing. By optimizing object and DNS allocations in an integrated fashion, ADA-OBJ/DNS further improves response time over ADA$\mathrm{OBJ}^{*}$ (see Figure 11).

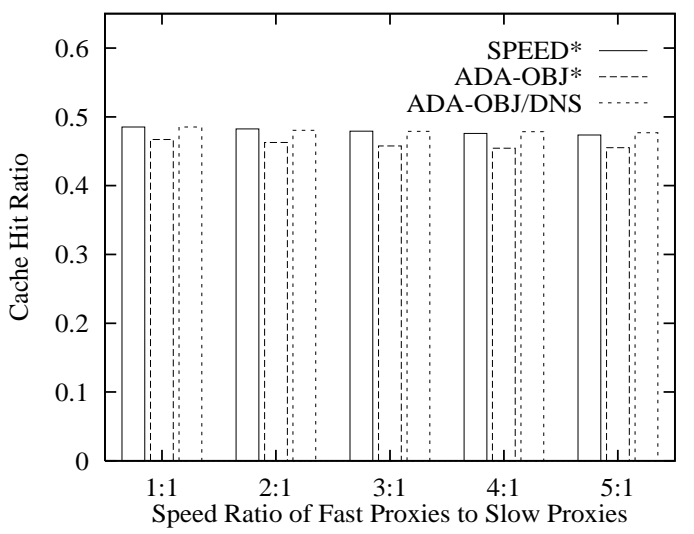

Figure 14: Cache Hit Ratio for Proxies of Different Speeds

An interesting point to note from Figure 11 is that ADA-OBJ* performs slightly worse than SPEED* when

\footnotetext{
${ }^{7}$ This conclusion also holds for SPEED and ADA-OBJ under static client configuration. However, the impact of heterogeneous speeds can be more clearly shown under dynamic client configuration where the skewness of original request distribution is reduced.
} 
all proxies have the same speed. The reason is that under dynamic client configuration, the client to proxy association changes frequently. The active clients that issue high volume of requests are associated with different proxies at different times. This leads to less stable arrival rate of original requests at each proxy, thereby reducing the effectiveness of ADA-OBJ* which adjusts the object allocation based on the distribution of request arrivals among the proxies.

Finally, we examine the impact of the time-to-live (TTL) value on hash routing performance. A lower TTL value means the domain name server has more control over the assignment of original requests. Figure 15 shows the results for the 3-1-1999 trace when the speed ratio between the fast and slow proxies was set at 4 . In general, the average response time decreases as the TTL period gets shorter. ADA-OBJ* and ADA-OBJ/DNS consistently outperform SPEED* for all the TTL values examined. Even when the TTL value is as low as 1 second, the adaptive schemes still maintain performance gain over SPEED*.

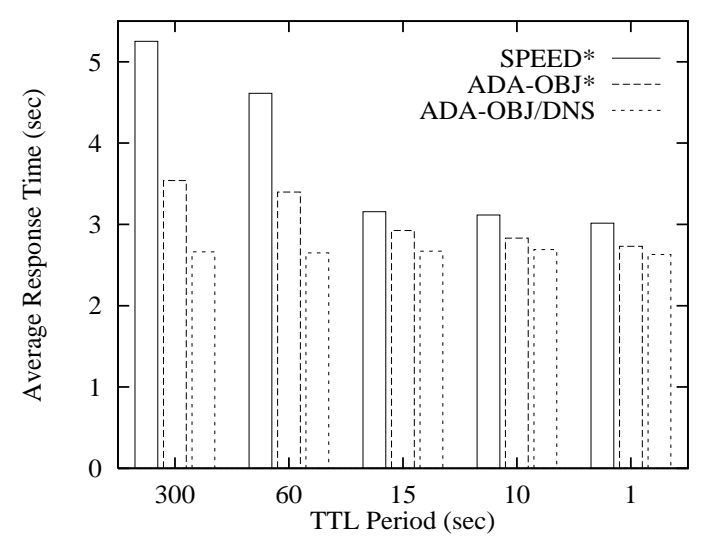

Figure 15: Average Response Time for Different TTL Values

\section{Related Work}

There is some existing work on hash routing for web proxies $[7,26,27,28]$. However, none of them specifically addressed the issue of dynamically allocating objects to the proxies to improve hash routing performance. Wu et $a l$. [26, 27] studied a controlled local replication scheme to reduce request response time for hash routing. This is different from our approach of dynamically adapting object allocation to the traffic pattern. Valloppillil et al. [8] and Karger et al. [28] developed a robust hashing scheme and a consistent hashing strategy respectively with the objective of minimizing the proportion of objects whose hashed proxies have to be changed when proxies are added or removed. Ross [7] further extended the robust hashing scheme to the case of heterogeneous proxies that have different processing speeds and storage capacities. These studies all assumed the object allocation strategy was given and did not propose any specific allocation strategy.

Some load balancing techniques have been proposed for distributed web servers [29, 30, 25, 31]. However, 
most of them did not consider the impact of request direction on caching effectiveness. For the same reason, early load sharing techniques developed for general purpose distributed systems [32, 33, 34, 35] are not suitable for web proxy clusters either. Moreover, the focus of the above schemes has been on balancing the load of the servers. While this may be sufficient when the servers have the same processing speed, it does not necessarily minimize the response time for speed-heterogeneous servers [14]. Harchol-Balter et al. [36] proposed a task assignment policy based on task sizes for distributed server systems. The size-based policy was shown to outperform sizeblind policies when task sizes were highly variable. Although the requirement of the knowledge of task sizes may be practical for a web site in which a cluster of web servers serve a fixed set of objects maintained by the content provider, it is not appropriate for a cluster of web proxies that dynamically retrieve objects from many different web sites based on user requests.

Pai et al. [37], Aron et al. [24, 38] and Bunt [39] investigated the integration of load balancing and effective caching to improve the performance of web servers. However, the proposed schemes need to maintain per-object status of the list of servers that store copies of an object in their caches. While the overhead introduced may be acceptable for a web site due to relatively low number of objects maintained by the site, it is too expensive for a cluster of web proxies that retrieve huge volume of objects from many web sites on behalf of the clients. Therefore, these schemes are not suitable for web proxy clusters. The hash routing scheme, on the other hand, associates objects with proxies in the form of a hash function, thereby involving little overhead to maintain the object-to-proxy mapping. In our previous paper [40], a preliminary study was done on optimizing object and DNS allocations to improve hash routing performance. However, the allocation strategies were not designed to dynamically adapt to current traffic pattern. The work reported in this paper studies adaptive hash routing schemes for different client configurations and shows that they significantly outperform the static speed-based hash routing scheme.

\section{Conclusion}

In this paper, we have investigated adaptive hash routing among a cluster of web proxies. A mathematical model is developed to analyze the steady-state performance of hash routing. Based on this model, the optimal hash routing problem is studied. Two adaptive hash routing schemes, ADA-OBJ and ADA-OBJ/DNS, are proposed for static and dynamic client configurations respectively. Object allocation is optimized in the static case, and both object and DNS allocations are optimized in the dynamic case.

Simulation experiments using actual proxy traces have been conducted to evaluate the proposed schemes. The results show that they significantly outperform the intuitive static hash routing scheme based only on the proxy speeds. It is found that: (1) even if there is no skewness in client to proxy association and object allocation, the workload distribution among the proxies can still be quite unbalanced, and adaptive hash routing can reduce the 
load imbalance; (2) the negative impact of skewed client to proxy association can be greatly reduced by properly adjusting the object allocation; (3) assigning web objects and mapping DNS queries to the proxies proportionally to their speeds does not produce the best performance when the proxies have different speeds; (4) it is desirable to optimize object and DNS allocations in an integrated fashion for dynamic client configuration.

\section{Acknowledgements}

The work described in this paper was supported by a grant from the Research Grants Council of the Hong Kong Special Administrative Region, China (RGC) (Project No. HKUST6066/00E), as well as a joint grant from RGC and the National Natural Science Foundation of China (Project No. N_HKUST618/02). The work was done when Xueyan Tang was with the Department of Computer Science, the Hong Kong University of Science and Technology.

\section{References}

[1] S. Glassman. A caching relay for the world wide web. Computer Networks and ISDN Systems, 27(2):165173, November 1994.

[2] C. Aggarwal, J. L. Wolf, and P. S. Yu. Caching on the world wide web. IEEE Transactions on Knowledge and Data Engineering, 11(1):94-107, January/February 1999.

[3] J. Shim, P. Scheuermann, and R. Vingralek. Proxy cache algorithms: Design, implementation, and performance. IEEE Transactions on Knowledge and Data Engineering, 11(4):549-562, July/August 1999.

[4] M. Rabinovich and O. Spatscheck. Web Caching and Replication. Addison-Wesley, 2002.

[5] X. Tang and S. T. Chanson. Coordinated en-route web caching. IEEE Transactions on Computers, 51(6):595-607, June 2002.

[6] A. Luotonen and K. Altis. World wide web proxies. Computer Networks and ISDN Systems, 27(2):147-154, November 1994.

[7] K. W. Ross. Hash routing for collections of shared web caches. IEEE Network, 11(6):37-44, November/December 1997.

[8] V. Valloppillil and K. W. Ross. Cache array routing protocol v1.0. Internet Draft, February 1998. http://ircache.nlanr.net/Cache/ICP/carp.txt.

[9] D. Wessels and K. Claffy. Internet Cache Protocol (ICP), version 2. RFC 2186, September 1997. 
[10] L. Fan, P. Cao, J. Almeida, and A. Z. Broder. Summary cache: A scalable wide-area web cache sharing protocol. IEEE/ACM Transactions on Networking, 8(3):281-293, June 2000.

[11] S. Gadde, M. Rabinovich, and J. Chase. Reduce, reuse, recycle: An approach to building large Internet caches. In Proceedings of the 6th Workshop on Hot Topics in Operating Systems (HOTOS), May 1997.

[12] WAP Forum. Wireless application protocol architecture specification. WAP-210-WAPArch-20010712-a, July 2001.

[13] S. Hadjiefthymiades and L. Merakos. Using proxy cache relocation to accelerate web browsing in wireless/mobile communications. In Proceedings of the 10th International World Wide Web Conference (WWW), pages 26-35, May 2001.

[14] X. Tang and S. T. Chanson. Optimizing static job scheduling in a network of heterogeneous computers. In Proceedings of the 29th International Conference on Parallel Processing (ICPP), pages 373-382, August 2000.

[15] D. G. Thaler and C. V. Ravishankar. Using name-based mappings to increase hit rates. IEEE/ACM Transactions on Networking, 6(1):1-14, February 1998.

[16] M. Garey and D. Johnson. Computers and Intractability: A Guide to the Theory of NP-Completeness. W. H. Freeman, 1979.

[17] L. Breslau, P. Cao, L. Fan, G. Phillips, and S. Shenker. Web caching and zipf-like distributions: Evidence and implications. In Proceedings of IEEE INFOCOM'99, pages 126-134, March 1999.

[18] M. F. Arlitt and C. L. Williamson. Internet web servers: Workload characterization and performance implications. IEEE/ACM Transactions on Networking, 5(5):631-645, October 1997.

[19] R. Jain. The Art of Computer Systems Performance Analysis: Techniques for Experimental Design, Measurement, Simulation, and Modeling. John Wiley \& Sons, 1991.

[20] L. Kleinrock. Queueing Systems, Volume I: Theory. John Wiley \& Sons, 1975.

[21] J. L. Zachary. Introduction to Scientific Programming: Computational Problem Solving Using Maple and C. TELOS/Springer-Verlag, 1996.

[22] Digital Equipment Corporation. Digital's web proxy traces. http://ftp.digital.com/pub/dec/traces/proxy/webtraces.htn 1996.

[23] J. Meadows. Boeing proxy logs. ftp://researchsmp2.cc.vt.edu/pub/boeing, 1999. 
[24] M. Aron, P. Druschel, and W. Zwaenepoel. Efficient support for P-HTTP in cluster-based web servers. In Proceedings of the 1999 USENIX Annual Technical Conference, pages 185-198, June 1999.

[25] M. Colajanni, P. S. Yu, and D. M. Dias. Analysis of task assignment policies in scalable distributed webserver systems. IEEE Transactions on Parallel and Distributed Systems, 9(6):585-600, June 1998.

[26] K.-L. Wu and P. S. Yu. Load balancing and hot spot relief for hash routing among a collection of proxy caches. In Proceedings of the 19th IEEE International Conference on Distributed Computing Systems (ICDCS), pages 536-543, June 1999.

[27] K.-L. Wu and P. S. Yu. Replication for load balancing and hot spot relief for proxy caches with hash routing. Distributed and Parallel Databases, 13(2):203-220, March 2003.

[28] D. Karger, A. Sherman, A. Berkheimer, B. Bogstad, R. Dhanidina, K. Iwamoto, B. Kim, L. Matkins, and Y. Yerushalmi. Web caching with consistent hashing. Computer Networks, 31(11-16):1203-1213, May 1999.

[29] E. D. Katz, M. Butler, and R. McGrath. A scalable HTTP server: The NCSA prototype. Computer Networks and ISDN Systems, 27(2):155-164, November 1994.

[30] D. M. Dias, W. Kish, R. Mukherjee, and R. Tewari. A scalable and highly available web server. In Proceedings of the 41st IEEE Computer Society International Conference (COMPCON), pages 85-92, February 1996.

[31] M. Colajanni and P. S. Yu. A performance study of robust load sharing strategies for distributed heterogeneous web servers. IEEE Transactions on Knowledge and Data Engineering, 14(2):398-414, March/April 2002.

[32] S. Zhou. A trace-driven simulation study of dynamic load balancing. IEEE Transactions on Software Engineering, 14(9):1327-1341, September 1988.

[33] O. Kremien and J. Kramer. Methodical analysis of adaptive load sharing algorithms. IEEE Transactions on Parallel and Distributed Systems, 3(6):747-760, November 1992.

[34] N. G. Shivaratri, P. Krueger, and M. Singhal. Load distribution for locally distributed systems. IEEE Computer, 8(12):33-44, December 1992.

[35] C.-C. Hui and S. T. Chanson. Hydrodynamic load balancing. IEEE Transactions on Parallel and Distributed Systems, 10(11):1118-1137, November 1999.

[36] M. Harchol-Balter, M. E. Crovella, and C. D. Murta. On choosing a task assignment policy for a distributed server system. Journal of Parallel and Distributed Computing, 59(2):204-228, November 1999. 
[37] V. S. Pai, M. Aron, G. Banga, M. Svendsen, P. Druschel, W. Zwaenepoel, and E. Nahum. Locality-aware request distribution in cluster-based network servers. In Proceedings of the 8th ACM International Conference on Architectural Support for Programming Languages and Operating Systems (ASPLOS), pages 205-216, October 1998.

[38] M. Aron, D. Sanders, P. Druschel, and W. Zwaenepoel. Scalable content-aware request distribution in cluster-based network servers. In Proceedings of the 2000 USENIX Annual Technical Conference, pages 323-336, June 2000.

[39] R. B. Bunt, D. L. Eager, G. M. Oster, and C. L. Williamson. Achieving load balance and effective caching in clustered web servers. In Proceedings of the 4th International Web Caching Workshop (WCW), April 1999.

[40] X. Tang and S. T. Chanson. Optimal hash routing for web proxies. In Proceedings of the 21th IEEE International Conference on Distributed Computing Systems (ICDCS), pages 191-198, April 2001.

\section{Biography}

Xueyan Tang received his BEng degree in computer science and engineering from Shanghai Jiao Tong University, Shanghai, China in 1998, and his PhD degree in computer science from the Hong Kong University of Science and Technology in 2003. He is currently an Assistant Professor in the School of Computer Engineering at Nanyang Technological University, Singapore. His research interests include web and Internet (particularly caching, replication and content delivery), mobile and pervasive computing (especially data management and delivery), peer-to-peer and sensor networks, streaming multimedia, and distributed systems.

Samuel T. Chanson received his $\mathrm{PhD}$ degree in Electrical Engineering and Computer Sciences from the University of California, Berkeley in 1975. He was a faculty member at the School of Electrical Engineering, Purdue University for two years before joining the Department of Computer Science at the University of British Columbia where he became a full professor and director of its Distributed Systems Research Group. He joined the Hong Kong University of Science and Technology in 1993.

Professor Chanson has been widely consulted by industry and government institutes in North America and Asia in Internet and communication technologies, and has served on the program committees of numerous international conferences on distributed systems and computer communications. He was the Conference Co-Chair of IEEE ICDCS in 1998 and IFIP FORTE/PSTV in 1999. He has served on the editorial boards of three international journals, including IEEE/ACM Transactions on Networking.

Professor Chanson's research interests include web technologies, multimedia communication, Internet security, and distributed systems. He has published more than 160 papers in the above areas. 